NSF-ITP-94-53

hep-th/9408005

July 1994

\title{
CRITICAL BEHAVIOR OF THE MARINARI-PARISI MODEL
}

\author{
Shyamoli Chaudhuri and Joseph Polchinski \\ Institute for Theoretical Physics \\ University of California \\ Santa Barbara, California 93106-4030
}

\begin{abstract}
We consider the continuum string theory corresponding to the Marinari-Parisi supersymmetric matrix model. We argue that the world-sheet physics is exotic, and different from any known supersymmetric string theory. The embedding superspace coordinates become disordered on the world-sheet, but because of the noncompactness of the embedding time the disorder becomes complete only at asymptotic world-sheet scales.
\end{abstract}


Understanding the nature of string theory beyond perturbation theory remains one of the key problems in fundamental theory. Matrix models are one of the few means of obtaining insight into this question, (1) having revealed, for example, the existence of unexpectedly large nonperturbative string effects [2]. Many important non-perturbative issues, especially the structure of the vacuum, are likely to involve spacetime supersymmetry in an essential way. A simple spacetime supersymmetric matrix model has been formulated by Marinari and Parisi[3], but there has been relatively little further development 4 , 5, 6, 7]. In particular, the continuum string theory which corresponds to the scaling limit of the Marinari-Parisi model has never been identified. In this paper we partially resolve this issue. The result is surprising, with rather exotic world-sheet physics, and it is different from any known supersymmetric string theory.

The Marinari-Parisi model[3] is the quantum mechanics of an $N \times N$ matrix in a one-dimensional superspace

$$
\boldsymbol{\Phi}(\tau, \theta, \bar{\theta})=\mathbf{M}(\tau)+\bar{\theta} \mathbf{\Psi}(\tau)+\overline{\mathbf{\Psi}}(\tau) \theta+\theta \bar{\theta} \mathbf{F}(\tau)
$$

The action ist

$$
S=-N \int d \tau d \bar{\theta} d \theta \operatorname{Tr}\left\{\frac{1}{2} \bar{D} \boldsymbol{\Phi} D \boldsymbol{\Phi}+W(\boldsymbol{\Phi})\right\},
$$

with $D=\partial_{\bar{\theta}}+\theta \partial_{\tau}, \bar{D}=-\partial_{\theta}-\bar{\theta} \partial_{\tau}$. The Feynman graph expansion for this model generates a discretization of random surfaces in superspace. Marinari and Parisi[3] solved the zero fermion number sector by diagonalizing $\mathbf{M}$ and rewriting the theory in terms of non-relativistic free fermions [8]. This was done for the cubic superpotential $W(\boldsymbol{\Phi})=\frac{1}{2} \boldsymbol{\Phi}^{2}-\frac{1}{3} \lambda \boldsymbol{\Phi}^{3}$, which is much simpler than the general polynomial case, and has been shown to have the generic critical behavior of this class of potentials [7]. Dabholkar [4] extended this to include those components of $\boldsymbol{\Psi}$ and $\overline{\boldsymbol{\Psi}}$ which are diagonal in the same basis as $\mathbf{M}$, and obtained a supersymmetric extension of the free fermi theory.

\footnotetext{
${ }^{1}$ For reviews, see ref. [1].

${ }^{2}$ We take the embedding time $\tau$ to be Euclidean, so that the world-sheet path integral is a convergent gaussian; Minkowski amplitudes are as usual defined by continuation.
} 
The model with cubic superpotential has a critical point at $\lambda_{c}^{2}=1 / 6 \sqrt{3}$, where the perturbation series diverges and large graphs dominate. In the double-scaling limit, $\lambda \rightarrow \lambda_{c}$ with the renormalized inverse string coupling, $\kappa^{-1}=N\left(\lambda_{c}-\lambda\right)^{5 / 2}$ held fixed, graphs of all topologies survive. The critical behavior is then expected to be described by some spacetime supersymmetric string theory. Various possibilities, and difficulties associated with each, are discussed in ref. [4. Rather than repeat these arguments here, we will point out a puzzle that has not previously been emphasized.

In order to obtain a nontrivial double-scaling limit, it is necessary[四] to rescale the embedding time, holding

$$
\tau\left(\lambda_{c}-\lambda\right)^{1 / 4}=\text { fixed }
$$

This is contrary to the situation in the bosonic $d=1$ theory [9], where the embedding time is not rescaled. Moreover, it would appear to violate general principles of quantum field theory: time translation is a global symmetry of the world-sheet theory, and so should not be renormalized 10].

To see what is happening, let us examine the expected continuum worldsheet theory. The superspace propagator for the theory (2) is

$$
\frac{1}{2}(\bar{D} D-D \bar{D}+2)\left(1-\partial_{\tau}^{2}\right)^{-1} \text {. }
$$

As usual in the matrix model, one expects the replacement of $\exp \left(\partial_{\tau}^{2}\right)$ for the propagator $\left(1-\partial_{\tau}^{2}\right)^{-1}$ to leave the theory in the same universality class, since their infra-red behavior coincides. The propagator between vertices at superspace positions $(\tau, \theta, \bar{\theta})$ and $\left(\tau^{\prime}, \theta^{\prime}, \bar{\theta}^{\prime}\right)$ becomes

$$
\exp \left\{-\frac{1}{2}\left[\tau^{\prime}-\tau-\left(\bar{\theta}^{\prime}-\bar{\theta}\right) \theta+\bar{\theta}\left(\theta^{\prime}-\theta\right)\right]^{2}+\left(\theta^{\prime}-\theta\right)\left(\bar{\theta}^{\prime}-\bar{\theta}\right)\right\} .
$$

The naive continuum limit gives the Euclidean action

$$
S=\frac{z_{1}}{2}\left(\partial_{a} \tau-\partial_{a} \bar{\theta} \theta+\bar{\theta} \partial_{a} \theta\right)^{2}-z_{2} \partial_{a} \theta \partial_{a} \bar{\theta},
$$

where we allow for general normalizations $z_{1}$ and $z_{2}$. This could also have been written down directly as the most relevant action allowed by the supersymmetry

$$
\delta \theta=\epsilon, \quad \delta \bar{\theta}=\bar{\epsilon}, \quad \delta \tau=-\epsilon \bar{\theta}-\bar{\epsilon} \theta .
$$


The action (6) is not free, and in fact is not conformally invariant. The one-loop beta functions can be computed as

$$
\mu \partial_{\mu} z_{1}=g^{2} z_{1} / \pi, \quad \mu \partial_{\mu} z_{2}=g^{2} z_{2} / \pi
$$

where the effective coupling $g^{2}=z_{1} / z_{2}^{2}$ satisfies $\mu \partial_{\mu} g^{2}=-2 g^{4} / \pi$. At longer world-sheet distances, the coupling grows and both $\tau$ and $\theta, \bar{\theta}$ become more disordered. There are two logical possibilities for the infrared limit: a nontrivial fixed point or a mass gap. In fact, we can largely exclude the former possibility. At a fixed point, one expects that the spacetime translation and supersymmetries, being global symmetries of the world-sheet theory, give rise to a current algebra,

$$
\begin{aligned}
j_{\theta}(z) j_{\bar{\theta}}(0) & \sim \frac{k}{z^{2}}+\frac{j_{\tau}(0)}{z} \\
j_{\tau}(z) j_{\theta}(0) & \sim j_{\tau}(z) j_{\bar{\theta}}(0) \sim \text { analytic. }
\end{aligned}
$$

However, taking the operator product of $j_{\tau}$ with (9) and using (10) implies that the $j_{\tau} j_{\tau}$ operator product is analytic. In a unitary theory, this would strictly imply that $j_{\tau}$ is trivial. In the present case the second order fermionic action is not unitary, but the argument still suggests that $j_{\tau}$ is trivial - that is, that there is a mass gap for world-sheet states with nonzero spacetime frequency. Since both beta functions (8) have the same sign, we expect that $\theta$ and $\bar{\theta}$ also develop a mass gap.

The rather surprising conclusion is that the spacetime supersymmetry of the matrix quantum mechanics problem does not survive in the critical theory: the superspace coordinates become massive, leaving only the worldsheet metric. This accounts for a key feature of the exact solution. The equal-time expectation values of the Marinari-Parisi model are the same as the expectation values in the $d=0$ bosonic string theory [3], both being given by

$$
\int d \boldsymbol{\Phi} e^{-\operatorname{Tr}\{W(\boldsymbol{\Phi})\}} \ldots .
$$

This equivalence now has the simple world-sheet explanation that these theories are actually the same at long world-sheet distances. 
This cannot, however, be the end of the story. By the rescaling (3) of $\tau$, Dabholkar [4] obtained a theory with non-trivial spacetime dynamics, including nonperturbative supersymmetry breaking. The rescaling, toward longer embedding-time scales as the critical point is approached, agrees in direction with our argument that $\tau$ is becoming disordered. But how can a non-trivial dynamics be consistent with a mass gap, since one would then expect all $\tau$-dependence to disappear from the critical theory? The key here is the unusual feature that the $\tau$-coordinate is non-compact. This is the only example of which we are aware in which a non-compact field disorders. For a compact field, there is some length scale at which the disorder becomes comparable to the range of the field and so one can say the field is completely disordered. For a non-compact field, however, one would expect that as one integrates to longer distances, the fluctations grow, but that they do not become infinite at any finite scale. Thus, by scaling to longer embedding time scales as the world-sheet cosmological canstant is taken to the critical point, one can obtain a non-trivial limit. In other words, for expectation values at fixed embedding time or frequency scales there is a world-sheet mass gap, but the gap goes to zero as the embedding time scale goes to zero.

Unfortunately we have not been able to make this picture more quantitative, and have not been able to derive the scaling exponent (3). The non-compactness of $\tau$ makes the theory (6) appear complicated even on a flat world-sheet, and we do not know of an analytic approach. But we believe that our general picture - that the superspace coordinates become disordered at long world-sheet distances, but the embedding time becomes fully disordered only asymptotically - is correct, both because of its plausibility, and because of the difficulty of understanding the rescaling (3) of $\tau$ in any other way.

In conclusion, the Marinari-Parisi model has exotic world-sheet and presumably also spacetime physics, but it is rather different from the usual spacetime supersymmetric string theories. Most of these involve world-sheet chirality. It is possible to introduce chirality into the matrix model vertices, and Siegel [11] has proposed a discretization of the Green-Schwarz super-

\footnotetext{
${ }^{3}$ Various heuristic arguments have all led us to the incorrect scaling $\tau \propto\left(\lambda_{c}-\lambda\right)^{-1 / 2}$.
} 
string, but we are not aware of a solvable example.

\section{Acknowledgements}

This work was supported in part by National Science Foundation grants PHY89-04035 and PHY91-16964. 


\section{References}

[1] P. Ginsparg and G. Moore, in Recent Directions in Particle Theory, Proceedings of the 1992 TASI, ed. J. Harvey and J. Polchinski (World Scientific, Singapore, 1993) hep-th/9304011;

I. Klebanov, in String Theory and Quantum Gravity, Proceedings of the Trieste School 1991, eds. J. Harvey et. al. (World Scientific, Singapore, 1992).

[2] S. Shenker, Proceedings of the Cargese Workshop on Random Surfaces, Quantum Gravity, and Strings (1990).

[3] E. Marinari and G. Parisi, Phys. Lett. B240 (1990) 375.

[4] A. Dabholkar, Nucl. Phys. B368 (1992) 293.

[5] M. Karliner and S. Migdal, Mod. Phys. Lett. A5 (1990) 2565;

S. Belluci, T. R. Govindrajan, A. Kumar, and R. N. Oerter, Phys. Lett. B249 (1990) 49;

J. González, Phys. Lett. B255 (1991) 367;

G. Ferretti, "The Untruncated Marinari-Parisi Superstring," Chalmers preprint ITP 93-41, hep-th/9310002.

[6] A. Jevicki and J. P. Rodrigues, Phys. Lett. B268 (1991) 53;

J. D. Cohn and H. Dykstra, Mod. Phys. Lett. A7 (1992) 1163;

R. Brustein, M. Faux, B. A. Ovrut, "Supersymmetric Field Theory from Supermatrix Models," CERN preprint CERN-TH-7353-94, hepth/9407164.

[7] J. P. Rodrigues and A. J. Van Tonder, Int. J. Mod. Phys. A8 (1993) 2517.

[8] E. Brézin, C. Itzykson, G. Parisi, and J.-B. Zuber, Comm. Math. Phys. 59 (1978) 35.

[9] D. J. Gross and N. Miljković, Phys. Lett. B238 (1990) 217;

E. Brézin, V. A. Kazakov, and A. B. Zamolodchikov, Nucl. Phys. B333 
(1990) 673;

P. Ginsparg and J. Zinn-Justin, Phys. Lett. B240 (1990) 333.

[10] K. Hepp, Comm. Math. Phys. 1 (1965) 95.

[11] W. Siegel, "Randomizing the Superstring," Stony Brook preprint ITPSB-94-08, hep-th/9403144. 